\title{
Invariant Measures in Quasi-Metric Spaces
}

\author{
M.V. Marchi
}

\begin{abstract}
We prove that in separable, complete, quasi-metric spaces there exists exactly one probability measure with bounded support, invariant with respect to given families of contractions and weights. The invariant measure has compact support and is an attractor in the space of probability measures with bounded support equipped with a metric defined in term of Hölder continuous functions.
\end{abstract}

Keywords: Quasi-metric spaces, invariant self-similar measures, Hölder continuous functions, fractals, multifractals

AMS subject classification: 28A80, 28A33, 28C5, 54E35

\section{Introduction}

In fractal theory we are interested in measures invariant with respect to finite families of functions $S_{1}, \ldots, S_{N}$ and weights $m_{1}, \ldots, m_{N}$, i.e. in measures $\mu$ such that

$$
\mu=\sum_{i=1}^{N} m_{i} \mu \circ S_{i}^{-1}
$$

where $m_{i}>0$ are constants such that $\sum_{i=1}^{N} m_{i}=1$.

Hutchinson [2] proved that when the functions $S_{i}$ are contractions in $\mathbb{R}^{n}$, there exists exactly one invariant probability measure $\mu$ with compact support. The support of $\mu$ is the compact set invariant with respect to the family $\left\{S_{1}, \ldots, S_{N}\right\}$, i.e. the unique non-empty compact set $K$ such that $K=\sum_{i=1}^{N} S_{i}(K)$. Moreover, $\mu$ is an attractor for the map

$$
T(\nu)=\sum_{i=1}^{N} m_{i} \nu \circ S_{i}^{-1}
$$

Maria Vittoria Marchi: Univ. degli Studi di Roma "La Sapienza", Dip. di Matem. "G. Castelnuovo", Piazzale Aldo Moro 2, 00185 Roma, Italy; marchi@mat.uniroma1.it

ISSN 0232-2064 / \$2.50 C Heldermann Verlag Berlin 
with respect to the weak convergence of probability measures with compact supports. For example, an $n$-dimensional cube $Q$, which is invariant with respect to $2^{n}$ similarities of contraction factor $2^{-1}$, supports the normalized restriction to the cube of the $n$-dimensional Lebesgue measure, that we denote by $\mu$. The latter is the unique probability measure on the cube invariant with respect to dilations and translations that make up the $2^{n}$ similarities. Furthermore, the sequence $\left(T^{n}(\nu)\right)=((T \circ \cdots \circ T)(\nu))$ converges weakly to $\mu$ for every probability measure $\nu$ with compact support.

It is natural to extend the fractal theory by replacing the space $\mathbb{R}^{n}$ with a quasi-metric space. Actually, these spaces occour, for example, in harmonic analysis (see Stein [9]) or in the dynamic fractal theory, when a self-similar set is endowed with the Lagrangian metric (see Mosco $[5,6]$ ).

When the space is of homogeneous type, the contractions are similarities of contraction factors $l_{i}$ and $m_{i}=l_{i}^{d_{f}}$, where $d_{f}$ is the fractal dimension, density arguments show that the normalized restriction to the invariant set of the $d_{f}$-dimensional Hausdorff measure is the unique invariant measure (see Marchi [4]).

In the Euclidean case, the existence and uniqueness of a probability measure, invariant with respect to given families of general contractions and weights, has been proved by using the fixed point theorem. Actually, once the space of probability measures with compact support is equipped with the metric

$$
\delta(\mu, \nu)=\sup \left\{\left|\int f d \mu-\int f d \nu\right|: f-\text { Lipschitz with constant } L_{f} \leq 1\right\},
$$

$T$ turns out to be a contraction. Moreover, the subspaces of probability measures with equibounded supports turn out to be complete. In fact, every Cauchy sequence in the metric $\delta$ defines a positive linear form on the family of Lipchitz maps. Due to density with respect to the uniform convergence on compacta of the latter family in the space $\mathcal{C}_{0}$ of continuous functions with compact support, the form turns out to be a positive linear functional on $\mathcal{C}_{0}$. Then, by the Riesz's representation theorem, it is an integral with respect to a probability measure, to which the Cauchy sequence turns out to converge in the metric $\delta$ and then weakly.

Mosco [7] proved that Hutchinson's results still hold in locally compact, quasi-metric spaces. In the quasi-metric case, the family of Lipschitz maps may be too poor to define a metric on the space of probability measures. Actually, by the presence of the constant $c_{T} \geq 1$ in the quasi-triangle inequality, the quasi-distance function can fail even to be continuous. Nevertheless, once in the metric $\delta$ the Lipschtz functions are replaced by the Hölder continuous functions of the same Hölder exponent of the Macias-Segovia quasi-metric 
(a locally Hölder continuous quasi-metric metrically equivalent to the original one), the set of probability measures with equibounded compact supports turns out to be a complete metric space.

In this paper we extend Mosco's results by proving that in a separable, complete, quasi-metric space there exists exactly one probability measure, with bounded support, invariant with respect to $T$. More precisely, we prove

Theorem. Let $S_{1}, \ldots, S_{N}$ be contractions in a separable, complete, quasimetric space $(X, d)$ and $m_{1}, \ldots, m_{N}$ be positive constants such that $\sum_{i=1}^{N} m_{i}=$ 1. Then:

(i) There exists exactly one probability measure $\mu$ with bounded support that satisfies (1.1). Moreover, supp $\mu=K$, where $K$ is the unique non-empty compact set, invariant with respect to $\left\{S_{1}, \ldots, S_{N}\right\}$.

(ii) For every probability measure $\nu$ with bounded support, the sequence $\left(T^{n}(\nu)\right)$ converges weakly to the measure $\mu$.

The plan of the paper is the following: In Section 2 we recall the DaniellStone theorem and its applications in metrizable spaces. In Section 3 we define in the space of probability measures with bounded support a metric in terms of Hölder continuous functions and prove that the convergence with respect to this metric is equivalent to the weak convergence. Finally, in Section 4 we prove our main result.

\section{Measures in metrizable spaces}

In this section we recall the Daniell-Stone theorem, its applications in metrizable spaces and some results on probability measures. For notations and proofs we refer to Bauer [1] and Parthasarathy [8].

Given a set $X$, a vector space $\mathcal{F}$ of real functions on $X$ and a positive linear form $I$ on $\mathcal{F}$, the Daniell-Stone theorem allows one to construct a measure $\mu$ in $X$, with respect to which $I$ and $\mathcal{F}$ turn out to be the integral and the family of elementary functions, respectively. Obviously, in order to apply the DaniellStone theorem, $\mathcal{F}$ and $I$ must satisfy the properties of the latter mathematical objects, i.e. $\mathcal{F}$ must be a Stone vector lattice and $I$ an abstract integral the definitions of which will be given below.

Definition 2.1. A Stone vector lattice on a set $X$ is a vector space $\mathcal{F}$ of real functions on $X$ such that

(i) $|u| \in \mathcal{F}$

(ii) $\inf (u, 1) \in \mathcal{F}$

for all $u \in \mathcal{F}$. 
When the Stone vector lattice contains the costant functions, condition (ii) follows from condition (i).

Definition 2.2. A set $G \subset X$ is said to be $\mathcal{F}$-open, if there exists an increasing sequence of positive functions $u_{n}$ in $\mathcal{F}$ such that $1_{G}=\sup u_{n}$.

The system of $\mathcal{F}$-open subsets of $X$ is closed with respect to countable unions. Further, $G$ is $\mathcal{F}$-open whenever, for a function $u \in \mathcal{F}, G=\{x \in X$ : $u(x)>0\}$. We denote by $\mathcal{A}(\mathcal{F})$ the smallest $\sigma$-algebra containing the $\mathcal{F}$-open subsets of $X$, and we say $\mathcal{A}(\mathcal{F})$ to be the $\sigma$-algebra generated by $\mathcal{F}$.

Definition 2.3. An abstract integral on a Stone vector lattice $\mathcal{F}$ is a linear form $I$ on $\mathcal{F}$ with the following properties:

(i) Positivity: $I(u) \geq 0$ for all $u>0$ in $\mathcal{F}$.

(ii) Monotonicity: for every sequence $\left(u_{n}\right)$ in $\mathcal{F}$ decreasing to 0 , inf $I\left(u_{n}\right)=$ 0 .

Theorem 2.4 (Daniell-Stone). Let $\mathcal{F}$ be a Stone vector lattice on a set $X$ and $I$ be an abstract integral on $\mathcal{F}$. Then:

(i) There exists exactly one measure $\mu$ on $\mathcal{A}(\mathcal{F})$ with the properties

$$
\begin{aligned}
\mathcal{F} & \subseteq L^{1}(\mu) \\
I(u) & =\int u d \mu \text { for every } u \in \mathcal{F} \\
\mu(E) & =\inf \{\mu(G): E \subseteq G, G \text { is } \mathcal{F}-\text { open }\} \text { for all } E \in \mathcal{A}(\mathcal{F}) .
\end{aligned}
$$

(ii) If $\mathcal{F}$ contains the constant functions, the measure $\mu$ is uniquely determined by properties (2.1) - (2.2) and is finite.

When $X$ is a metrizable space, the $\sigma$-algebra of Borel sets $\mathcal{B}(X)$, i.e. the smallest $\sigma$-algebra containing the open subsets of $X$, coincides with the $\sigma$ algebra of Baire sets $\mathcal{A}\left(\mathcal{C}_{b}\right)$, where $\mathcal{C}_{b}$ denotes the Stone vector lattice of all bounded continuous functions on $X$. Therefore, every abstract integral on $\mathcal{C}_{b}$ is an integral with respect to a finite Borel measure. Conversely, since every integral with respect to a finite Borel measure is an abstract integral on $\mathcal{C}_{b}$, we can consider abstract integrals on $\mathcal{C}_{b}$ and finite Borel measures as the same mathematical objects.

Moreover, by (2.3) it follows that every finite Borel measure $\mu$ is Borelregular, i.e.

$$
\mu(A)=\left\{\begin{array}{l}
\inf \{\mu(G): A \subseteq G \text { with } G \text { open }\} \\
\sup \{\mu(F): F \subseteq A \text { with } F \text { closed }\}
\end{array}\right.
$$

for every $A \in \mathcal{B}(X)$. By taking into account the identity between finite Borel measures and abstract integrals on $\mathcal{C}_{b}$, we define in the space of probability 
measures $\mathcal{M}^{1}=\mathcal{M}^{1}(X)$ with

$$
\mathcal{M}^{1}=\{\text { Borel measures in } X \text { with bounded support and unitary mass }\}
$$

the weak topology. We recall that a sequence $\left(\mu_{n}\right)$ in $\mathcal{M}^{1}$ is said to be weakly convergent to a measure $\mu \in \mathcal{M}^{1}$ if $\lim _{n \rightarrow+\infty} \int f d \mu_{n}=\int f d \mu$ for every $f \in \mathcal{C}_{b}$.

Sometimes it is convenient to consider the probability measures as abstract integrals on Stone vector lattices, rich enough to generate $B(X)$, but smaller than $\mathcal{C}_{b}$. For example, when $X$ is locally compact and $\mathcal{C}_{0}$ is the Stone vector lattice of continuous functions with compact support, any positive linear form on $\mathcal{C}_{0}$ is continuous, once $\mathcal{C}_{0}$ has been equipped with the uniform convergence on compacta, and then, by Dini's theorem, monotone. Therefore, by the Daniell-Stone theorem, it is a Radon measure, i.e. a Borel measure, finite on compacta and regular, i.e it satisfies (2.4) and $\mu(A)=\sup \{\mu(K): K \subseteq$ $A$ with $K$ compact\}.

Another case in which we consider the probability measures as abstract integrals on Stone vector lattices smaller than $\mathcal{C}_{b}$ is in metrizing $\mathcal{M}^{1}$. When $X$ is separable, we can construct in $\mathcal{M}^{1}$ a metric whose topology coincides with the weak one (see [8: Theorem 6.2]). This metric depends on the topology on $X$, but, in general, does not reflect the properties of a given distance $d$ in $X$. In order to define in $\mathcal{M}^{1}$ a metric which takes into account the distance $d$, we consider the probabilty measures as abstract integrals on the Stone vector lattice $\operatorname{Lip}(X)$ of bounded functions, Lipschitz continuous with respect to $d$. Due to the Lipschitz continuity of $d$, this lattice is rich enough to generate $\mathcal{B}(X)$. Thus every abstract integral on $\operatorname{Lip}(X)$ is an integral with respect to a finite Borel measure and viceversa.

In the next section the topology on the metrizable space $X$ will be defined in terms of a quasi-distance $d$. We recall that a quasi-distance $d$ satisfies the properties of a distance with the triangle inequality replaced by the quasitriangle inequality: there exists a constant $c_{T} \geq 1$ such that

$$
d(x, z) \leq c_{T}(d(x, y)+d(y, z)) \text { for all } x, y, z \in X
$$

In this case $\mathcal{C}_{b}$ will be replaced by the Stone vector lattice

$$
C_{b}^{0, \gamma}=\left\{\begin{array}{l}
\text { bounded functions, Hölder continuous } \\
\text { of exponent } \gamma \text { with respect to } d
\end{array}\right\}
$$

where $\gamma$ is as in Theorem 3.1. We will prove that, when $X$ is separable, $\mathcal{A}\left(\mathcal{C}_{b}^{0, \gamma}\right)=\mathcal{B}(X)$ and, by using $\mathcal{C}_{b}^{0, \gamma}$, we will define a metric $\delta$ in $\mathcal{M}^{1}$. In order 
to prove the completeness of $\left(\mathcal{M}^{1}, \delta\right)$, we will need to prove that a bounded positive linear form $L$ on $\mathcal{C}_{b}^{0, \gamma}$, obtained as limit of $\mu_{n}$-integrals, where $\left(\mu_{n}\right)$ is a Cauchy sequence in $\left(\mathcal{M}^{1}, \delta\right)$, is an abstract integral. By using the density on compacta of $\mathcal{C}_{b}^{0, \gamma}$ in $\mathcal{C}_{0}$, Mosco [7] proved that, when $X$ is locally compact and the measures have compact support, the positive linear form $L$ is an abstract integral.

In the following section, without assuming $X$ to be locally compact, we will prove the monotonicity of $L$ by showing that any Cauchy sequence in $\left(\mathcal{M}^{1}, \delta\right)$ is uniformly tight. We recall that a sequence $\left(\mu_{n}\right)$ is said to be uniformly tight if for every $\eta>0$ there exists a compact set $K_{\eta}$ such that, for each $n>0$, $\mu_{n}\left(K_{\eta}\right)>1-\eta$.

In order to prove that the weak convergence and the convergence with respect to the metric $\delta$ are equivalent, we will use the following theorem.

Theorem 2.5 [8: Theorem 6.8]. Let $X$ be a complete, separable, metrizable space and $\left(\mu_{n}\right)$ be a sequence in $\mathcal{M}^{1}$. Then $\left(\mu_{n}\right)$ converges weakly to a measure $\mu \in \mathcal{M}^{1}$ if and only if

$$
\lim _{n \rightarrow+\infty} \sup \left\{\left|\int f d \mu_{n}-\int f d \mu\right|: f \in \mathcal{A}_{0}\right\}=0
$$

for every family $\mathcal{A}_{0}$ of functions equicontinuous at all points $x \in X$ and uniformly bounded.

\section{A metric on the set of Borel measures in quasi-metric spaces}

A quasi-distance $d$ on a set $X$ is a function $d: X \times X \rightarrow \mathbb{R}^{+}$such that:

(i) $d(x, y) \geq 0$ for all $x, y \in X$

(ii) $d(x, y)=0$ if and only if $x=y$

(iii) $d(x, y)=d(y, x)$ for all $x, y \in X$

(iv) For a constant $c_{T}=c_{T}^{d} \geq 1, d(x, z) \leq c_{T}(d(x, y)+d(y, z))$ for all $x, y, z \in X$.

For every $x \in X$ and $R>0$ we set

$$
\begin{aligned}
& B^{d}(x, R)=\{y \in X: d(x, y)<R\} \\
& C^{d}(x, R)=\{y \in X: d(x, y) \leq R\}
\end{aligned}
$$


Further, for $x_{0} \in X, E \subseteq X$ and $\varepsilon>0$ we set

$$
\begin{aligned}
d_{x_{0}}(x) & =d\left(x, x_{0}\right) \\
d_{E}(x) & =\inf \{d(x, a): a \in E\} \\
I_{\varepsilon}^{d}(E) & =\left\{x \in X: d_{E}(x)<\varepsilon\right\} \\
\operatorname{diam}^{d} E & =\inf \{d(x, y): x, y \in E\} .
\end{aligned}
$$

A quasi-metric space is a topological space $X$ on which a quasi-distance $d$ is defined, such that the balls $B^{d}(x, R)$ form a basis of neighborhoods of $x$ for the topology of $X$. This space turns out to be a uniform space whose uniformity has a countable base. Then, by the Alexandroff-Uryson theorem, it is metrizable. In particular, any subset of $X$ is complete if and only if it is sequentially complete, and it is compact if and only if it is complete and totally bounded.

In order to introduce a metric in the space of probability measures on $X$, i.e. in $\mathcal{M}^{1}$ with respect to which a map $T: \mathcal{M}^{1} \rightarrow \mathcal{M}^{1}$, defined in term of contractions on $(X, d)$, turns out to be a contraction, we need to find a Stone vector lattice of continuous functions $\mathcal{F}$ which takes into account the metric properties of $(X, d)$ and that is rich enough so that $\mathcal{A}(\mathcal{F})=\mathcal{B}(X)$. When $d$ is a metric, the seeked lattice is that of Lipschitz maps. Actually, in this case for every $E \subseteq X$ the function $d_{E}(x)$ is a Lipschitz map. Because of the presence of the constant $c_{T}$ in the quasi-triangle inequality, in a quasi-metric space $d_{x_{0}}$ can even fail to be continuous. Unfortunately, we cannot replace $d$ with the distance given by the Alexandroff-Uryson theorem, because the latter is not metrically equivalent to $d$.

The following Theorem 3.1 shows that there exists a quasi-distance $\tilde{d}$, metrically equivalent to $d$, such that $\tilde{d}_{x_{0}}$ is locally Hölder continuous. We recall that a real function $f$ on a quasi-metric space $X$ is said to be Hölder continuous of exponent $\gamma, 0<\gamma \leq 1$, if there exists a constant $k>0$ such that

$$
|f(x)-f(y)| \leq k d(x, y)^{\gamma} \text { for all } x, y \in X .
$$

The constant

$$
|f|_{0, \gamma}^{d}=\inf \left\{k>0:|f(x)-f(y)| \leq k d(x, y)^{\gamma} \text { for every } x, y \in X\right\}
$$

is called the Hölder constant of $f$. Further, the function $f$ is said to be locally Hölder continuous of exponent $\gamma$ if, for every closed bounded $C \subset X$, there exists a constant $k=k(C)>0$ such that

$$
|f(x)-f(y)| \leq k d(x, y)^{\gamma} \text { for all } x, y \in C .
$$


Moreover, we recall that two quasi-distances $d$ and $\tilde{d}$ on $X$ are said to be metrically equivalent if they satisfy (3.1).

Obviously, a function $f$ is Hölder continuous of exponent $\gamma$ with respect to a quasi-distance $d$ if and only if it is Hölder continuous of exponent $\gamma$ with respect to a quasi-distance $\tilde{d}$ metrically equivalent to $d$. The unique difference consists in the Hölder constants which differ by a constant independent of $f$.

Theorem 3.1 [3]. Let d be a quasi-distance on a set $X$. Then there exists a quasi-distance $\tilde{d}$ on $X$ and constants $c_{1}>0, c_{2}, c$ and $0<\gamma \leq 1$ depending only on $c_{T}$ such that

$$
\begin{gathered}
c_{1} d(x, y) \leq \tilde{d}(x, y) \leq c_{2} d(x, y) \\
|\tilde{d}(x, z)-\tilde{d}(y, z)| \leq c(\tilde{d}(x, z)+\tilde{d}(y, z))^{1-\gamma} \tilde{d}(x, y)^{\gamma}
\end{gathered}
$$

for every $x, y, z \in X$.

By the previous theorem it follows in particular that, for every $x_{0} \in X$ and $R>0, B^{\tilde{d}}(x, R)$ and $I_{\varepsilon}^{\tilde{d}}(E)$ are open, $C^{\tilde{d}}(x, R)$ is closed and $\overline{B^{\tilde{d}}(x, R)} \subseteq$ $C^{\tilde{d}}(x, R)$. Because of its regularity, in the following we will consider $\tilde{d}$ instead of $d$ as a quasi-metric on $X$, and we will omit to write $\tilde{d}$ when the balls, the diameters, the quasi-triangle and the Hölder constants are referred to $\tilde{d}$.

Let $\mathcal{C}_{b}^{0, \gamma}$ be the set of bounded Hölder continuous functions of exponent $\gamma$ on $(X, \tilde{d})$. It is easy to prove that $\mathcal{C}_{b}^{0, \gamma}$ is a Stone vector lattice of continuous functions which contains the constants. We will prove that $\mathcal{C}_{b}^{0, \gamma}$ is large enough so that $\mathcal{A}\left(\mathcal{C}_{b}^{0, \gamma}\right)=\mathcal{B}(X)$.

We remark that for fixed $x_{0} \in X$ and $r>0, B\left(x_{0}, r\right)=\{x \in X$ : $\left.r-f_{r}(x)>0\right\}$, where

$$
f_{r}(x)=\inf \left(r, \tilde{d}_{x_{0}}(x)\right) .
$$

Therefore, to prove that the $B\left(x_{0}, r\right)$ is $\mathcal{C}_{b}^{0, \gamma}$-open it suffices to prove that $f_{r} \in \mathcal{C}_{b}^{0, \gamma}$. The latter result follows from Lemma 3.2 below, that we prove for sake of completeness.

Lemma 3.2 [7]. Let $x_{0} \in X$, and let $r>0$ be such that there exists $y_{0} \in X$ with $\tilde{d}\left(x_{0}, y_{0}\right)=r$. Then, for every $x \in B\left(x_{0}, r\right)$ and $y \in X$ with $\tilde{d}\left(y, x_{0}\right)>r$, there exists $z \in B\left(x_{0}, c_{T}\left(1+2 c_{T}\right) r\right)$ such that $r \leq \tilde{d}\left(z, x_{0}\right)$ and $\tilde{d}(z, x) \leq \tilde{d}(y, x)$.

Proof. Let $x \in B\left(x_{0}, r\right)$. For every $u \in C\left(x_{0}, r\right), \tilde{d}(u, x)<2 c_{T} r$, so

$$
C\left(x_{0}, r\right) \subset B\left(x, 2 c_{T} r\right) .
$$

Moreover, for every $v \in B\left(x, 2 c_{T} r\right), \tilde{d}\left(v, x_{0}\right)<c_{T}\left(1+2 c_{T}\right) r$, thus

$$
B\left(x, 2 c_{T} r\right) \subset B\left(x_{0}, c_{T}\left(1+2 c_{T}\right) r\right) .
$$


Let $y \in X$. If $\tilde{d}\left(y, x_{0}\right)<c_{T}\left(1+2 c_{T}\right) r$, we choose $z=y$, and if $\tilde{d}\left(y, x_{0}\right) \geq$ $c_{T}\left(1+2 c_{T}\right) r$, we choose $z=y_{0}$. Actually, by $(3.4), \tilde{d}(y, x) \geq 2 c_{T} r$ whereas, by $(3.3), \tilde{d}\left(y_{0}, x\right)<2 c_{T} r$

Lemma 3.3. Let $x_{0} \in X$ and let $r>0$ be such that there exists $y_{0} \in X$ with $\tilde{d}\left(x_{0}, y_{0}\right)=r$. Then $f_{r} \in \mathcal{C}_{b}^{0, \gamma}$.

Proof. We have

$$
\begin{aligned}
& \left|f_{r}(x)-f_{r}(y)\right| \\
& \quad= \begin{cases}0 & \text { if } \tilde{d}\left(x, x_{0}\right), \tilde{d}\left(y, x_{0}\right) \geq r \\
\left|\tilde{d}\left(x, x_{0}\right)-\tilde{d}\left(y, x_{0}\right)\right| \leq c(2 r)^{1-\gamma} \tilde{d}(x, y)^{\gamma} & \text { if } x, y \in B\left(x_{0}, r\right) .\end{cases}
\end{aligned}
$$

When $x \in B\left(x_{0}, r\right)$ and $\tilde{d}\left(y, x_{0}\right) \geq r$, by Lemma 3.2 there exists $z \in B\left(x_{0}, c_{T}(1+\right.$ $\left.2 c_{T}\right) r$ ) such that

$$
\begin{aligned}
0 & <f_{r}(y)-f_{r}(x) \\
& =r-\tilde{d}\left(x, x_{0}\right) \\
& \leq \tilde{d}\left(z, x_{0}\right)-\tilde{d}\left(x, x_{0}\right) \\
& \leq c\left(\tilde{d}\left(z, x_{0}\right)+\tilde{d}\left(x, x_{0}\right)\right)^{1-\gamma} \tilde{d}(x, z)^{\gamma} \\
& <c\left(c_{T}\left(1+2 c_{T}\right) r+r\right)^{1-\gamma} \tilde{d}(x, z)^{\gamma} \\
& \leq c\left(c_{T}\left(1+2 c_{T}\right) r+r\right)^{1-\gamma} \tilde{d}(x, y)^{\gamma}
\end{aligned}
$$

and the statement is proved

Lemma 3.4. For every $x_{0} \in X$ and $r>0$, the ball $B\left(x_{0}, r\right)$ is $\mathcal{C}_{b}^{0, \gamma}$-open.

Proof. By Lemma 3.3, $B\left(x_{0}, r\right)$ is $\mathcal{C}_{b}^{0, \gamma}$-open whenever there exists $y_{0} \in X$ with $\tilde{d}\left(x_{0}, y_{0}\right)=r$. Otherwise, or $B\left(x_{0}, r\right)$ is countable union of $\mathcal{C}_{b}^{0, \gamma}$-open balls $B\left(x_{0}, r_{n}\right)$ where, for every $0<r_{n}<r$, there exists $y_{n} \in X$ with $\tilde{d}\left(x_{0}, y_{n}\right)=r_{n}$ and then it is $\mathcal{C}_{b}^{0, \gamma}$-open, or $B\left(x_{0}, r\right)=\left\{x_{0}\right\}$. In this case we can assume without loss of generality $r \leq 1$. Thus $f_{r} \in \mathcal{C}_{b}^{0, \gamma}$ since

$$
\left.\mid f_{r}(x)-f_{(} y\right) \mid= \begin{cases}0 & \text { when } x, y \neq x_{0} \text { or } x=y \\ r \leq r^{\gamma} \leq \tilde{d}(x, y)^{\gamma} & \text { when } x=x_{0} \text { and } y \neq x_{0}\end{cases}
$$

and the lemma is proved

Proposition 3.5. Let $(X, d)$ be a separable, quasi-metric space. Then $\mathcal{A}\left(\mathcal{C}_{b}^{0, \gamma}\right)=\mathcal{B}(X)$.

Proof. From the separability of the space it follows that any open subset of $X$ is the countable union of open balls and then $\mathcal{C}_{b}^{0, \gamma}$-open 
Set

$$
\delta(\mu, \nu)=\sup \left\{\left|\int f d \mu-\int f d \nu\right|: f \in \mathcal{C}_{b}^{0, \gamma} \text { with }|f|_{0, \gamma} \leq 1\right\} .
$$

Theorem 3.6. Let $(X, d)$ be a separable, quasi-metric space. Then $\left(\mathcal{M}^{1}, \delta\right)$ is a metric space.

Proof. For any $\mu, \nu \in \mathcal{M}^{1}, \delta(\mu, \nu) \leq 2 R^{\gamma}$ where $R=\operatorname{diam}(\operatorname{supp} \mu \cup$ $\operatorname{supp} \nu)$. Actually, for any fixed $f \in \mathcal{C}_{b}^{0, \gamma}$ with $|f|_{0, \gamma} \leq 1$ and $x_{0} \in \operatorname{supp} \mu \cup$ $\operatorname{supp} \nu$

$$
\begin{array}{rl}
\mid \int f & d \mu-\int f d \nu \mid \\
& =\left|\int\left(f-f\left(x_{0}\right)\right) d \mu-\int\left(f-f\left(x_{0}\right)\right) d \nu\right| \\
& \leq \int \tilde{d}\left(x, x_{0}\right)^{\gamma} d \mu+\int \tilde{d}\left(x, x_{0}\right)^{\gamma} d \nu \\
\leq & 2 R^{\gamma} .
\end{array}
$$

Obviously, for every $\mu, \nu, \sigma \in \mathcal{M}^{1}, 0 \leq \delta(\mu, \nu)=\delta(\nu, \mu)$ and $\delta(\mu, \nu) \leq \delta(\mu, \sigma)+$ $\delta(\sigma, \nu)$. If $\delta(\mu, \nu)=0$, then $\int f d \mu=\int f d \nu$ for every $f \in \mathcal{C}_{b}^{0, \gamma}$. Thus $I(f)=$ $\int f d \mu\left(=\int f d \nu\right)$ is an abstract integral on $\mathcal{C}_{b}^{0, \gamma}$. Therefore, by Theorem 2.4 and Proposition 3.5, $\mu=\nu$ on $\mathcal{A}\left(\mathcal{C}_{b}^{0, \gamma}\right)=\mathcal{B}(X)$

Let $\left(\mu_{n}\right)$ be a Cauchy sequence in $\left(\mathcal{M}^{1}, \delta\right)$. For every $f \in \mathcal{C}_{b}^{0, \gamma},\left(\int f d \mu_{n}\right)$ is a Cauchy sequence in $\mathbb{R}$. Actually, $\int f d \mu_{n}=|f|_{0, \gamma} \int \phi d \mu_{n}$ where $\phi=$ $\left(|f|_{0, \gamma}\right)^{-1} f$. Let us define

$$
L(f)=\lim _{n \rightarrow+\infty} \int f d \mu_{n}
$$

As $\left(\mu_{n}\right)$ is a Cauchy sequence in $\left(\mathcal{M}^{1}, \delta\right)$,

$$
\lim _{n \rightarrow+\infty} \sup \left\{\left|\int f d \mu_{n}-L(f)\right|: f \in \mathcal{C}_{b}^{0, \gamma} \text { and }|f|_{0, \gamma} \leq 1\right\}=0 .
$$

We will prove that $\left(\mu_{n}\right)$ converges in $\left(\mathcal{M}^{1}, \delta\right)$ by showing that $L$ is an abstract integral on $\mathcal{C}_{b}^{0, \gamma}$, so that, by the Daniell-Stone theorem, there exists $\mu \in \mathcal{M}^{1}$ such that $L(f)=\int f d \mu$. Therefore, by $(3.5), \mu_{n}$ converges to $\mu$.

From the linearity and the positivity of the integral and the limit it follows that $L$ is a positive linear functional on $\mathcal{C}_{b}^{0, \gamma}$. In order to prove the monotonicity of $L$ we will prove that $\left(\mu_{n}\right)$ is uniformly tight whenever $X$ is separable and bounded. 
Lemma 3.7. Let $(X, d)$ be a bounded quasi-metric space. Then, for every $E \subseteq X, \tilde{d}_{E} \in \mathcal{C}_{b}^{0, \gamma}$. Moreover, $\left|\tilde{d}_{E}\right|_{0, \gamma} \leq c(2 R)^{1-\gamma}$, where $R=\operatorname{diam} X$.

Proof. Let $x, y \in X$ and $\varepsilon>0$, and let us assume $\tilde{d}_{E}(x) \geq \tilde{d}_{E}(y)$. Then, by choosing $a=a_{\varepsilon}(y) \in E$ such that $\tilde{d}(y, a) \leq \tilde{d}_{E}(y)+\varepsilon$, we have

$$
\tilde{d}_{E}(x)-\tilde{d}_{E}(y) \leq \tilde{d}(x, a)-\tilde{d}(y, a)+\varepsilon .
$$

By Theorem 3.1 it follows

$$
\begin{aligned}
\left|\tilde{d}_{E}(x)-\tilde{d}_{E}(y)\right| & \leq c(\tilde{d}(x, a)+\tilde{d}(y, a))^{1-\gamma} \tilde{d}(x, y)^{\gamma}+\varepsilon \\
& \leq c(2 R)^{1-\gamma} \tilde{d}(x, y)^{\gamma}+\varepsilon .
\end{aligned}
$$

By the arbitrariness of $\varepsilon,\left|\tilde{d}_{E}(x)-\tilde{d}_{E}(y)\right| \leq c(2 R)^{1-\gamma} \tilde{d}(x, y)^{\gamma}$

Remark. When $X$ is bounded, we can prove Lemma 3.5 and then Lemma 3.6 without assuming $X$ to be separable. By Lemma 3.7, every open $A \neq X$ is $\mathcal{C}_{b}^{0, \gamma}$-open. Actually, $A=\left\{x \in X: \tilde{d}_{X \backslash A}(x)>0\right\}$. Moreover $X$ is $\mathcal{C}_{b}^{0, \gamma}$-open as well, since the constant functions belong to $\mathcal{C}_{b}^{0, \gamma}$. Then $\mathcal{A}\left(\mathcal{C}_{b}^{0, \gamma}\right)=\mathcal{B}(X)$. By proceeding as in Theorem 3.6, we can prove that $\delta$ is a metric on $\mathcal{M}^{1}$.

Lemma 3.8. Let $(X, d)$ be a bounded, separable, complete quasi-metric space and let $\left(\mu_{n}\right)$ be a Cauchy sequence in $\left(\mathcal{M}^{1}, \delta\right)$. Then $\left(\mu_{n}\right)$ is uniformly tight.

Proof. Let $\left(x_{i}\right)$ be a dense sequence of points in $X$. We start by proving that, for each $\varepsilon>0$ and $\eta>0$, there exists $k>0$ such that, for any $n>0$,

$$
\mu_{n}\left(S_{k, \varepsilon}\right)>1-\eta
$$

where $S_{k, \varepsilon}=\bigcup_{i=1}^{k} C\left(x_{i}, \varepsilon\right)$.

Let $R=\operatorname{diam} X$. For any fixed $\zeta>0$ choose $\bar{n}=\bar{n}(\zeta)$ such that $\delta\left(\mu_{n}, \mu_{m}\right) \leq \frac{\zeta^{2}}{c(2 R)^{1-\gamma}}$ for every $m, n \geq \bar{n}$. By the separability of $X$ there exists $k(\zeta, \bar{n})>0$ such that $\mu_{\bar{n}}\left(S_{k(\zeta, \bar{n}), \zeta}\right)>1-\zeta$. The function

$$
f(x)=1-\frac{1}{\zeta} \inf \left(\zeta, \tilde{d}_{S_{k(\zeta, \bar{n}), \zeta}}(x)\right)
$$

is Hölder continuous of exponent $\gamma$ with Hölder constant $|f|_{0, \gamma} \leq \frac{c(2 R)^{1-\gamma}}{\zeta}$. Moreover, $0 \leq f(x) \leq 1$ for every $x \in X$ and

$$
f(x)= \begin{cases}0 & \text { when } x \notin S_{k(\zeta, \bar{n}), 2 c_{T} \zeta} \\ 1 & \text { when } x \in S_{k(\zeta, \bar{n}), \zeta}\end{cases}
$$


since $I_{\zeta}\left(S_{k(\zeta, \bar{n}), \zeta}\right) \subseteq S_{k(\zeta, \bar{n}), 2 c_{T} \zeta}$. Therefore, for every probability measure $\mu$,

$$
\mu\left(S_{k(\zeta, \bar{n}), \zeta}\right) \leq \int f d \mu \leq \mu\left(S_{k(\zeta, \bar{n}), 2 c_{T} \zeta}\right) .
$$

Then $\mu_{n}\left(S_{k(\zeta, \bar{n}), 2 c_{T} \zeta}\right)>1-2 \zeta$ for any $n \geq \bar{n}$. Actually,

$$
\begin{aligned}
1-\zeta & \leq \mu_{\bar{n}}\left(S_{k(\zeta, \bar{n}), \zeta}\right) \\
& \leq \int f d \mu_{\bar{n}} \\
& \leq \int f d \mu_{n}+\left|\int f d \mu_{\bar{n}}-\int f d \mu_{n}\right| \\
& \leq \mu_{n}\left(S_{k(\zeta, \bar{n}), 2 c_{T} \zeta}\right)+|f|_{0, \gamma} \frac{\zeta^{2}}{c(2 R)^{1-\gamma}} \\
& \leq \mu_{n}\left(S_{k(\zeta, \bar{n}), 2 c_{T} \zeta}\right)+\zeta .
\end{aligned}
$$

Set $k_{\zeta}=\max \left\{k(\zeta, \bar{n}), k_{1}(\zeta), \ldots, k_{\bar{n}-1}(\zeta)\right\}$, where $\mu_{n}\left(S_{k_{n}(\zeta), 2 c_{T} \zeta}\right)>1-2 \zeta$ for every $n<\bar{n}$. Then, for every $n>0$,

$$
\mu_{n}\left(S_{k_{\zeta}, 2 c_{T} \zeta}\right)>1-2 \zeta .
$$

For fixed $\varepsilon>0$ and $\eta>0,(3.6)$ follows from (3.7) by setting $\zeta=\min \left\{\frac{\varepsilon}{2 c_{T}}, \frac{\eta}{2}\right\}$. Select for each $j$ the integer $k_{j}$ such that $\mu_{n}\left(S_{k_{j}, \frac{1}{j}}\right)>1-\frac{\eta}{j}$ for all $n>0$. Due to the completeness of $X$,

$$
K_{\eta}=\bigcap_{j}\left(\bigcup_{i=1}^{k_{j}} C\left(x_{i}, \frac{1}{j}\right)\right)
$$

is compact since it is totally bounded and closed. Moreover, for each $n>0$, $\mu_{n}\left(K_{\eta}\right)>1-\eta$

Let $g$ be a bounded function on $X$ and $E \subseteq X$. We set $\|g\|_{E}=\sup \{|g(x)|$ : $x \in E\}$ and $\|g\|=\|g\|_{X}$.

Corollary 3.9. Let $(X, d)$ be a bounded, separable, complete quasi-metric space and let $\left(\mu_{n}\right)$ be a Cauchy sequence in $\left(\mathcal{M}^{1}, \delta\right)$. Then $L$ is an abstract integral on $\mathcal{C}_{b}^{0, \gamma}$.

Proof. We need only to prove the monotonocity of $L$. Let $\left(g_{k}\right)$ be a sequence in $\mathcal{C}_{b}^{0, \gamma}$ decreasing to 0 . For a fixed $\eta>0$ choose $K_{\eta}$ such that $\mu_{n}\left(K_{\eta}\right)>1-\eta$ for every $n>0$. Then, for every $n>0$,

$$
\begin{aligned}
\int g_{k} d \mu_{n} & =\int_{K_{\eta}} g_{k} d \mu_{n}+\int_{X-K_{\eta}} g_{k} d \mu_{n} \\
& \leq\left\|g_{k}\right\|_{K_{\eta}} \mu_{n}\left(K_{\eta}\right)+\left\|g_{1}\right\| \mu_{n}\left(X-K_{\eta}\right) \\
& \leq\left\|g_{k}\right\|_{K_{\eta}}+\left\|g_{1}\right\| \eta .
\end{aligned}
$$


As by the Dini theorem $\left(\left\|g_{k}\right\|_{K_{\eta}}\right)$ converges to 0 , we can choose $k(\eta)>0$ such that $\left\|g_{k}\right\|_{\mid K_{\eta}}<\eta$ whenever $k>k(\eta)$. Consequently, $\int g_{k} d \mu_{n} \leq\left(1+\left\|g_{1}\right\|\right) \eta$ for every $n>0$ and $k>k(\eta)$. By passing to the limit with respect to $n$ we obtain $L\left(g_{k}\right) \leq\left(1+\left\|g_{1}\right\|\right) \eta$ for every $k>k(\eta)$. Therefore $L\left(g_{k}\right)$ converges to 0 I

Theorem 3.10. Let $(X, d)$ be a bounded, separable, complete quasi-metric space. Then $\left(\mathcal{M}^{1}, \delta\right)$ is a complete metric space.

Proof. Let $\left(\mu_{n}\right)$ be a Cauchy sequence in $\left(\mathcal{M}^{1}, \delta\right)$. By Corollary $3.9, L$ is an abstract integral on $\mathcal{C}_{b}^{0, \gamma}$. Therefore, by Proposition 3.5 and Theorem 2.4, there exists exactly one finite Borel measure $\mu$ such that

$$
\int f d \mu=L(f)=\lim _{n \rightarrow+\infty} \int f d \mu_{n} \text { for every } f \in \mathcal{C}_{b}^{0, \gamma} .
$$

From (3.5) it follows that $\mu_{n}$ converges to $\mu$ with respect to the metric $\delta$. Furthermore, $\mu$ is a probability measure. Actually, by $(2.2), \mu(X)=L\left(1_{X}\right)=$ $\lim _{n \rightarrow+\infty} \int 1_{X} d \mu_{n}=1$

By the well known characterization of weak precompactness (see, e.g., [8: Theorem 6.7]) it follows that a Cauchy sequence in $\left(\mathcal{M}^{1}, \delta\right)$ converges weakly. For sake of completeness we prove the latter result.

Theorem 3.11. Let $(X, d)$ be a bounded, separable, complete quasi-metric space and let the sequence $\left(\mu_{n}\right)$ converge in $\left(\mathcal{M}^{1}, \delta\right)$ to a measure $\mu$. Then $\left(\mu_{n}\right)$ converges weakly to $\mu$.

Proof. Let us assume that $\left(\mu_{n}\right)$ does not converge weakly to $\mu$. Then there exist $f \in \mathcal{C}_{b}, \eta>0$ and a subsequence $\mu_{n_{k}}$ such that, for any $k>0$, $\left|\int f d \mu_{n_{k}}-\int f d \mu\right|>\eta$. By Lemma 3.8 there exists a compact set $K$ such that, for any $k>0, \mu_{n_{k}}(K)>1-\|f\|^{-1} \frac{\eta}{8}$. As by the Stone-Weierstrass theorem $\mathcal{C}_{b}^{0, \gamma}$ is dense in $\mathcal{C}_{b}$ with respect to the uniform convergence on compacta, there exists $g \in \mathcal{C}_{b}^{0, \gamma}$ such that $\|g-f\|_{K}<\frac{\eta}{8}$. By choosing $\bar{n}>0$ such that $\delta\left(\mu_{n}, \mu\right)<\left(|g|_{0, \gamma}\right)^{-1} \frac{\eta}{2}$, for every $n>\bar{n}$ we have, for every $n_{k}>\bar{n}$,

$$
\begin{aligned}
\eta & <\left|\int f d \mu_{n_{k}}-\int f d \mu\right| \\
& =\left|\int_{X-K} f d \mu_{n_{k}}-\int_{X-K} f d \mu+\int_{K} f d \mu_{n_{k}}-\int_{K} f d \mu\right| \\
& \leq \frac{\eta}{4}+\left|\int_{K}(g-f) d \mu_{n_{k}}-\int_{K}(g-f) d \mu\right|+\left|\int_{K} g d \mu_{n_{k}}-\int_{K} g d \mu\right| \\
& <\frac{\eta}{2}+\delta\left(\mu_{n_{k}}, \mu\right)|g|_{0, \gamma} \\
& <\eta
\end{aligned}
$$

and this contradiction proves our statement 
By Theorem 2.5 it follows that the converse still holds. Actually, $\{f \in$ $\left.\left.\mathcal{C}_{b}^{0, \gamma}:|f|_{0, \gamma}\right) \leq 1\right\}$ is a family of functions equicontinuous at all points and equibounded whenever $X$ is bounded. Therefore the weak convergence and the convergence with respect to the metric $\delta$ turn out to be equivalent when $(X, d)$ is a bounded, separable, complete quasi-metric space.

\section{Invariant measures in quasi-metric spaces}

In this section we will assume $(X, d)$ to be a separable, complete, quasi-metric space and $\tilde{d}$ to be the locally Hölder continuous quasi-metric given by Theorem 3.1 .

Definition 4.1. A map $S: X \rightarrow X$ is said to be a contraction in $(X, d)$ (of contraction factor $l$ ) if there exists a constant $0<l=l_{S}^{d}<1$ such that $d(S(x), S(y)) \leq l d(x, y)$ for all $x, y \in X$.

Let $S_{1}, \ldots, S_{N}$ be contractions in $(X, d)$ with contraction factors $l_{1}, \ldots, l_{n}$ and let $l=\max \left\{l_{1}, \ldots, l_{N}\right\}$. For every non-empty bounded set $A \subseteq X$, set

$$
\mathcal{S}(A)=\bigcup_{i=1}^{N} S_{i}(A)
$$

and by $\mathcal{S}^{n}$ denote the $n$-iterated map $\mathcal{S} \circ \cdots \circ \mathcal{S}$, i.e.

$$
\mathcal{S}^{n}(A)=\bigcup_{i_{1}, \ldots, i_{n}=1}^{N} S_{i_{1}, \ldots, i_{n}}(A)
$$

where $S_{i_{1} \ldots, i_{n}}=S_{i_{1}} \circ \cdots \circ S_{i_{n}}$ turn out to be contractions of contraction factors $l_{i_{1} \ldots i_{n}}=l_{i_{1}} \cdots l_{i_{n}}$.

We say that $A \subseteq X$ is invariant with respect to $\left\{S_{1}, \cdots, S_{N}\right\}$ or, briefly, with respect to $\mathcal{S}$, if $\mathcal{S}(A)=A$.

Let $m_{1}, \ldots, m_{N}$ be positive costants such that $\sum_{i=1}^{N} m_{i}=1$. For every $\mu \in \mathcal{M}^{1}$, set

$$
T \mu(E)=\sum_{i=1}^{N} m_{i} \mu \circ S_{i}^{-1}
$$

and by $T^{n}$ denote the $n$-iterated map $T \circ \cdots \circ T$, i.e.

$$
T^{n} \mu(E)=\sum_{i_{1}, \ldots, i_{n}=1}^{N} m_{i_{1}} \cdots m_{i_{n}} \mu \circ S_{i_{1}, \ldots, i_{n}}^{-1} .
$$


A Borel measure $\mu$ on $X$ is said to be invariant with respect to $\left\{S_{1}, \ldots, S_{N}\right\}$ and $\left\{m_{1}, \ldots, m_{N}\right\}$ or, briefly, with respect to $T$, if $T \mu=\mu$.

Before proving our main theorem we recall some results about contractions in quasi-metric spaces. For the proofs we refer to [4]. For $A$ and $B$ non-empty bounded subsets of $X$, define

$$
h(A, B)=\max \left\{\sup _{a \in A} \tilde{d}_{B}(a), \sup _{b \in B} \tilde{d}_{A}(b)\right\} .
$$

Let $H(X)=\{A \subset X: A$ non-empty and compact $\}$.

Proposition 4.2. $(H(X), h)$ is a complete quasi-metric space.

We start by considering the case in which $S_{1}, \ldots, S_{n}$ are contractions in $(X, \tilde{d})$.

Proposition 4.3. $\mathcal{S}$ is a contraction in $(H(X), h)$ of contraction factor $l$.

By Propositions 4.2 and 4.3 and the fixed point theorem in quasi-metric spaces, there exists exactly one non-empty compact set $K$, invariant with respect to $\mathcal{S}$. Moreover, $K$ is an attractor for $\mathcal{S}$, i.e. for every $C \in H(X)$ the sequence $h\left(\mathcal{S}^{n}(C), K\right)$ converges to 0 .

As in the metric space, we can prove the uniqueness of the invariant set also in the class of bounded closed subsets of $X$.

Lemma 4.4. Let $S$ be a contraction in $(X, \tilde{d})$ and $B \subset X$ be a nonempty, bounded set such that $S(B) \subseteq B$. Let $r>0$ and $A \subset \bigcup_{x \in B} C(x, r)$. Then $\overline{S^{n}(A)} \subset \bigcup_{x \in B} C\left(x, l^{n} r\right)$.

By the previous lemma it follows

Theorem 4.5. Let $\left\{S_{1}, \ldots, S_{N}\right\}$ be a family of contractions in $(X, \tilde{d})$. Then:

(i) There exists exactly one non-empty, bounded, closed set $K$, invariant with respect to $\mathcal{S}$. Namely, $K$ is compact.

(ii) For every non-empty bounded set $A \subset X$, the sequence $h\left(\overline{\mathcal{S}^{n}(A)}, K\right)$ converges to 0 .

Lemma 4.6. Let $\mu \in \mathcal{M}^{1}$. Let $S$ be a contraction on $(X, \tilde{d})$ and let $\mu \circ S^{-1}$ be the image of $\mu$ under $S$. Then $\operatorname{supp} \mu \circ S^{-1}=\overline{S(\operatorname{supp} \mu)}$.

Proof. We start by proving that $S(\operatorname{supp} \mu) \subseteq \operatorname{supp} \mu \circ S^{-1}$. Let $x_{0} \in$ $\operatorname{supp} \mu$. Then, for every $\varepsilon>0$, there exists $x_{\varepsilon}$ such that $\tilde{d}\left(x_{0}, x_{\varepsilon}\right)<\varepsilon$ and $\mu\left(B\left(x_{\varepsilon}, r\right)>0\right.$ for every $r>0$. Then $\tilde{d}\left(S\left(x_{0}\right), S\left(x_{\varepsilon}\right)\right)<l \varepsilon$ and $\mu \circ$ $S^{-1}\left(B\left(S\left(x_{\varepsilon}\right), l r\right)\right)>0$ since $B\left(x_{\varepsilon}, r\right) \subseteq S^{-1}\left(B\left(S\left(x_{\varepsilon}\right), l r\right)\right)$. Therefore, $S\left(x_{0}\right) \in$ $\operatorname{supp} \mu \circ S^{-1}$.

Conversely, let $x_{0} \notin \overline{S(\operatorname{supp} \mu)}$ and let $r>0$ be such that $B\left(x_{0}, r\right) \subset X \backslash$ $\overline{S(\operatorname{supp} \mu)}$. Then $S^{-1}\left(B\left(x_{0}, r\right)\right) \subset X \backslash \operatorname{supp} \mu$. Therefore, $x_{0} \notin \operatorname{supp} \mu \circ S^{-1}$ 
For $r>0$, set $X^{\prime}=\bigcup_{x \in K} C(x, r)$.

Proposition 4.7. $T$ is a contraction on $\left(\mathcal{M}^{1}\left(X^{\prime}\right), \delta\right)$.

Proof. Let $\mu \in \mathcal{M}^{1}\left(X^{\prime}\right)$. By Lemma $4.6 \operatorname{supp} T \mu=\bigcup_{i=1}^{N} \overline{S_{i}(\operatorname{supp} \mu)}$. Then by Lemma $4.4 \operatorname{supp} T \mu \subseteq X^{\prime}$. Let $\mu$ and $\nu \in \mathcal{M}^{1}\left(X^{\prime}\right)$. For every $f \in \mathcal{C}_{b}^{0, \gamma}$ with $|f|_{0, \gamma} \leq 1$, let $f_{i}=l^{-\gamma} f \circ S_{i}$. We have

$$
\begin{aligned}
\left|f_{i}(x)-f_{i}(y)\right| & =l^{-\gamma}\left|f\left(S_{i}(x)\right)-f\left(S_{i}(y)\right)\right| \\
& \leq l^{-\gamma} \tilde{d}^{\gamma}\left(S_{i}(x), S_{i}(y)\right) \\
& \leq\left(\frac{l_{i}}{l}\right)^{\gamma} \tilde{d}^{\gamma}(x, y) \\
& \leq \tilde{d}^{\gamma}(x, y) .
\end{aligned}
$$

Thus $f_{i} \in \mathcal{C}_{b}^{0, \gamma}$ and $\left|f_{i}\right|_{0, \gamma} \leq 1$. Then

$$
\begin{aligned}
\left|\int f d T \mu-\int f d T \nu\right| \leq \sum_{i=1}^{N} m_{i}\left|\int f \circ S_{i} d \mu-\int f \circ S_{i} d \nu\right| \\
\quad=\sum_{i=1}^{N} m_{i} l^{\gamma}\left|\int f_{i} d \mu-\int f_{i} d \nu\right| \\
\leq \sum_{i=1}^{N} m_{i} l^{\gamma} \delta(\mu, \nu) \\
=l^{\gamma} \delta(\mu, \nu) .
\end{aligned}
$$

Therefore $\delta(T \mu, T \nu) \leq l^{\gamma} \delta(\mu, \nu)$

As $X^{\prime}$ is bounded, by Theorem $3.10\left(\mathcal{M}^{1}\left(X^{\prime}\right), \delta\right)$ is complete. Therefore, by applying the fixed point theorem, we can prove that there exists exactly one probability measure $\mu \in \mathcal{M}^{1}\left(X^{\prime}\right)$, invariant with respect to $T$. By the arbitrariness of $r>0$ it follows that $\mu$ is unique in $\mathcal{M}^{1}$. Moreover, for every $\nu \in \mathcal{M}^{1}$, we can prove $\lim _{n \rightarrow+\infty} \delta\left(T^{n}(\nu), \mu\right)=0$ by applying the fixed point theorem to the contraction $T$ in the space $\left(\mathcal{M}^{1}\left(X^{\prime}\right), \delta\right)$, where $r>0$ is chosen large enough so that $\operatorname{supp} \nu \subseteq X^{\prime}$. Therefore, by Theorem 3.11, $T^{n}(\nu)$ converges weakly to $\mu$. Finally, by Lemma 4.6 and Theorem 4.5/(ii), $\operatorname{supp} \mu=K$.

We have proved

Theorem 4.8. Let $(X, d)$ be a separable complete quasi-metric space, $S_{1}, \ldots, S_{N}$ be contractions in $(X, \tilde{d})$ and $m_{1}, \ldots, m_{N}$ be positive constants such that $\sum_{i=1}^{N} m_{i}=1$. Then: 
(i) There exists exactly one probability measure $\mu$ with bounded support, invariant with respect to $T$. Moreover, $\operatorname{supp} \mu=K$, where $K$ is the unique non-empty compact set, invariant with respect to $\mathcal{S}$.

(ii) For every probability measure $\nu$ with bounded support, the sequence $T^{n}(\nu)$ converges weakly to the measure $\mu$.

We now extend our results to contractions in $(X, d)$.

Theorem 4.9. Let $(X, d)$ be a separable, complete, quasi-metric space, $S_{1}, \ldots, S_{N}$ be contractions in $(X, d)$ and let $m_{1}, \ldots, m_{N}$ be positive constants such that $\sum_{i=1}^{N} m_{i}=1$. Then:

(i) There exists exactly one compact set $K \subseteq X$ invariant with respect to $\mathcal{S}$ and one probability measure $\mu$, with bounded support, invariant with respect to $T$. Moreover, $\operatorname{supp} \mu=K$ and $K$ is compact.

(ii) For every probability measure $\nu$ with bounded support the sequence $T^{n}(\nu)$ converges weakly to the measure $\mu$.

Proof. Although the contractions $S_{i}$ might not be contractions in $(X, \tilde{d})$, when $n>\frac{\log c_{1}-\log c_{2}}{\log l}$ the maps $S_{i_{1} \ldots, i_{n}}$ turn out to be contractions in $(X, \tilde{d})$. Actually, $l_{i_{1} \ldots, i_{n}}^{\tilde{d}} \leq(l)^{n} \frac{c_{2}}{c_{1}}$. Then, by Theorem 4.8 , there exist exactly one nonempty compact set $K$ and one probability measure $\mu$ with bounded support that are the fixed points of $\mathcal{S}^{n}$ and $T^{n}$. By applying $\mathcal{S}$ and $T$ in the equalities $\mathcal{S}^{n}(K)=K$ and $T^{n}(\mu)=\mu$, we prove that $\mathcal{S}(K)$ and $T(\mu)$ are also fixed points of $\mathcal{S}^{n}$ and $T^{n}$. By uniqueness, $\mathcal{S}(K)=K$ and $T(\mu)=\mu$.

Acknowledgement. The author would like to thank Prof. Umberto Mosco for many helpful discussions.

\section{References}

[1] Bauer, H.: Probability Theory and Elements of Measure Theory. New York: Holt, Rinehart and Winston Inc. 1972.

[2] Hutchinson, J. E.: Fractal and self-similarity. Indiana Univ. Math. J. 30 (1981), $713-747$.

[3] Macias, R. A. and Segovia C.: Lipschitz functions on spaces of homogeneous type. Adv. Math. 33 (1979), $257-270$.

[4] Marchi, M. V.: Self-similarity in spaces of homogeneous type. Adv. Math. Sci. \& Appl. 9 (1999), $851-870$.

[5] Mosco, U.: Lagrangian metrics on fractals. Amer. Math. Soc. Proc. Symp. Appl. Math. 54 (1998), $301-323$.

[6] Mosco, U.: Lagrangian metrics and fractal dynamics. Proc. Int. Conf. Fractal Geometry and Stochastics II, University of Greifswald/Germany (eds.: C. Bandt et al.; Progr. Probab.: Vol. 46). Basel: Birkhäuser Verlag 2000. 
[7] Mosco, U.: Self-similar measures in quasi-metric spaces. Recent Trends in Nonlinear Analysis (Progr. Nonlinear Diff. Equ. and Appl.: Vol. 40). Basel: Birkhäuser Verlag 2000, pp. 233 - 348.

[8] Parthasarathy, K. R.: Probability Measures in Metric Spaces. London: Acad. Press 1967.

[9] Stein, E.: Harmonic Analysis. Princeton Univ. Press 1993.

Received 21.05.2002 